\title{
Avaliação da redução de matéria orgânica recalcitrante em lixiviado de aterro sanitário através da combinação dos processos de coagulação/floculação e de adsorção em carvão ativado em pó
}

\section{Evaluation of reduction of recalcitrant organic matter in landfill leachate by the combined process of coagualation/ floculation and powdered activated carbon adsorption}

\author{
Data de entrada: \\ 05/01/2016 \\ - Data de aprovação: \\ $25 / 05 / 2016$
}

\section{RESUMO}

O objetivo deste estudo foi avaliar o uso combinado de Carvão Ativado em Pó (CAP) com coagulante (cloreto férrico) na remoção de compostos orgânicos recalcitrantes presentes no lixiviado de aterro sanitário. Os resultados demonstraram que a aplicação de CAP como pós-tratamento ao processo de coagulação/floculação mostra-se mais vantajosa do que quando $\mathrm{d}$ em conjunto ao coagulante. Foram obtidos, para dosagem de 0,6 gFe.L $\mathrm{L}^{-1}$ e 4 gCAP.L ${ }^{-1}$, valores de remoção em torno de $96 \%$ para compostos orgânicos recalcitrantes, expressos em termos de carbono orgânico dissolvido (COD), e de 99,9\% para cor e turbidez.

Palavras-chave: carvão ativado em pó, matéria orgânica recalcitrante, lixiviado de aterro sanitário.

\section{ABSTRACT}

This study aimed to evaluate the combined use of Powdered Activated Carbon (PAC) with coagulant (ferric chloride) in the removal of recalcitrant organic compounds from landfill leachates.

The results showed that using PAC post-treatment to the coagulantion/floculation process was more advantageous than when PAC was dosed with coagulant. The recalcitrant organic compounds removal achieved expressed in terms of dissolved organic carbon (DOC) was around $96 \%$ with a dose of 0,6 gFe. $\mathrm{L}^{-1}$ and $4 \mathrm{gPAC} . \mathrm{L}^{-1}$, and removal of 99,9\% for color and turbidity.

Keywords: powdered activated carbon, recalcitrant organic compounds, landfill leachates.

Fábio Campos - Doutor em Ciências pela Faculdade de Saúde Pública da USP. Técnico responsável pelo Lab. de Saneamento "Prof. Lucas Nogueira Garcez" - Departamento de Engenharia Hidráulica e Ambiental da Escola Politécnica da USP; Prof. do Curso de Gestão Ambiental da Escola de Artes e Ciências Humanas - USP Leste. Endereço: Rua Kalil Mikail, 92 - Parque Paulistano/São Paulo, Brasil - CEP: 08080-440 - Fone (011)30915444 - e-mal: fcamposausp.br.

Roque Passos Piveli - Prof. Titular, Chefe do Depto. de Eng. Hidráliulica e Ambiental da Escola Politécnica da USP. 


\section{INTRODUÇÃO}

Lixiviados de aterro sanitário são definidos como efluentes líquidos gerados pela decomposição físico-química e biológica dos resíduos depositados em aterros sanitários, os quais são transferidos para uma fase líquida em função da percolação pela água de chuva, bem como da umidade natural de tais resíduos, gerando uma matriz aquosa de extrema complexidade (Christensen et al., 2001; Kjeldsen et al., 2002).

Tais efluentes apresentam concentração de matéria orgânica (biodegradáveis e não biodegradáveis - recalcitrantes) variável ao longo do tempo de funcionamento do aterro sanitário, podendo apresentar, por exemplo, uma variação de DQOO (Demanda Química de Oxigênio) da ordem de 1000 mg.L-1 (aterro "velho" - estabilizado) a 60.000 mg.L-1 (aterro "jovem”), onde as substâncias húmicas constituem um importante grupo, assim como outros componentes, tais como os compostos nitrogenados, metais pesados e sais inorgânicos (Campos, 2014; Renou et al., 2007; Moravia et al., 2006; Wiszniowiski et al., 2005; Çeçen e Çakiroglu, 2001; Q Qasim e Chiang, 1994).

A tratabilidade do lixiviado de aterro sanitário depende, portanto, da sua composição, assim como da característica do tipo de matéria orgânica presente (biodegradável ou recalcitrante), havendo diferentes tecnologias, incluindo processos biológicos, tratamento físico-químico, processo oxidativo avançado (POA), entre outros (Kurniawan et al., 2005).

Processos físico-químicos são indicados no tratamento de lixiviados estabilizados provenientes de células de aterros sanitário com idade de operação avançada, uma vez que os processos biológicos de tratamento não apresentam grandes eficiências na remoção dos compostos orgânicos remanescentes (recalcitrantes) (Li et al., 2010; Kurniawan et al., 2005).
A literatura apresenta diversos trabalhos referentes ao uso dessas tecnologias. Silva (2011), tratando lixiviado estabilizado com a adição de 400 mg. $\mathrm{L}^{-1}$ de $\mathrm{Fe}^{+3}$ juntamente com 2 mg. $\mathrm{L}^{-1}$ de polímero em $\mathrm{pH} 4,0$, conseguiu reduções da ordem de 98\% para cor e $80 \%$ para COD (Carbono Orgânico Dissolvido). Wiszniowski et al. (2005) indicam a remoção de DQQ e COD na ordem de 10 a 25\% em lixiviados novos, e de 50 a $60 \%$ em lixiviados estabilizados, mediante o uso de coagulantes. Tatsi (2003) cita outros trabalhos dos mesmos pesquisadores, onde se obtém eficiências de remoção de DQO em cerca de $75 \%$ em lixiviados estabilizados, contra remoção de 25 a 38\% em lixiviados novos. Kurniawan et al. (2005) citam, também, trabalho do grupo de pesquisa de Tatsi, tratando lixiviado estabilizado proveniente do Aterro de Thessaloniki - Grécia, onde, através de ajustes de $\mathrm{pH}$ e com adição de 1,5 g.L-1 de $\mathrm{FeCl}_{3}$, obtiveram remoção de 80\% de DQO. Wiszniowski et al. (2006) comentam que a aplicação de adsorção em carvão permite a remoção de 50 a $70 \%$ de $\mathrm{DQO}$ e $\mathrm{N}-\mathrm{NH}_{4}{ }^{+}$. Kurniawan et al. (2005) apresentam resultados de remoção da ordem de $90 \%$ para DQQ , reforçando sua aplicabilidade, sobretudo, na redução de compostos orgânicos recalcitrantes (não biodegradáveis).

Nesse contexto, o objetivo geral do estudo foi avaliar a possibilidade de se otimizar a remoção de compostos orgânicos recalcitrantes presentes no lixiviado proveniente de aterro sanitário estabilizado (expressos em termos de carbono orgânico dissolvido) por meio de aplicação combinada e sequencial dos processos de coagulação/floculação e de adsorção com Carvão Ativado em Pó (CAP).

\section{MATERIAIS E MÉTODOS}

\subsection{Características do Lixiviado de Aterro Sanitário}

Com o intuito de atender ao escopo deste estudo, foi utilizado nos testes lixiviado proveniente do Aterro Sanitário Sítio São João. 
O referido aterro situa-se na Zona Leste da capital paulista, na Estrada Sapopemba km 33 e, atualmente, encontra-se desativado. Durante os anos de 2004 e 2009, o aterro foi operado pela concessionária Ecourbis, recebendo diariamente cerca de 6000 toneladas de resíduos sólidos e gerando por volta de $1800 \mathrm{~m}^{3} \cdot \mathrm{d}^{-1}$ de lixiviado.

O lixiviado coletado foi devidamente caracterizado nas seguintes variáveis: COD, DQO, DBO, nitrogênio total kjedahl (NTK), nitrogênio amoniacal, sólidos em suspensão totais, cor aparente, turbidez, condutividade elétrica e pH. Os métodos analíticos foram empregados de acordo com o Standard Methods for the Examination of Water and Wastewater, $21^{\text {th }}$ Edition (APHA, 2005).

Dessa forma, para a realização dos ensaios físico-químicos, foram coletados $80 \mathrm{~L}$ desse lixiviado e acondicionados num único tanque a fim de garantir a homogeneidade de suas características ao longo do estudo.

\subsection{Delineamento dos ensaios de Coagulação/ Floculação}

Os ensaios de coagulação e floculação foram realizados mediante o uso de um equipamento de Jar Test convencional equipado com seis jarros, com volume de $500 \mathrm{~mL}$ de amostra. 0 procedimento experimental consistiu em três etapas:

- Coagulação: mistura rápida, a 180 rpm por seis minutos (Gradiente de velocidade $=300 \mathrm{~s}^{-1}$ );

- Floculação: mistura lenta a 50 rpm por 15 minutos (Gradiente de velocidade $=30 \mathrm{~s}^{-1}$ );

- Sedimentação: sem mistura por uma hora.

Após o período de sedimentação, o sobrenadante de cada jarro era retirado com auxílio de uma pipeta de $100 \mathrm{~mL}$ e transferido para um béquer, onde foram separados em duas alíquotas: uma denominada Amostra Decantada, e a outra, filtrada em papel de filtro de $20 \mu \mathrm{m}$, denominada de Amostra Filtrada, a fim de serem analisadas posteriormente.
Os experimentos foram conduzidos com variação de pH (de 3 a 9) em diferentes dosagens de coagulante (cloreto férrico - de 0,2 a 0,8 $\mathrm{gFe} . \mathrm{L}^{-1}$ ), sendo utilizado $\mathrm{HCl}$ ou $\mathrm{NaOH}$ para eventuais ajustes de $\mathrm{pH}$.

\subsection{Delineamento dos Experimentos de Adsorção}

Os experimentos de adsorção foram realizados em Jar Test com adição de Carvão Ativado em Pó (CAP) da marca BRASCARBO, de forma conjunta e subsequentemente ao processo de coagulação/ floculação. Em ambas as situações, variou-se a dosagem de CAP de 2 a $12 \mathrm{~g} . \mathrm{L}^{-1}$, e de coagulante (cloreto férrico), de 0,2 a 0,8 gFe. $\mathrm{L}^{-1}$, com tempo de contato de 3 horas a $150 \mathrm{rpm}$ (Gradiente de velocidade de $180 \mathrm{~s}^{-1}$ ) em $\mathrm{pH}$ pré-definido de acordo com os resultados obtidos com o ensaio de coagulação/floculação, seguidos de uma hora de sedimentação. O sobrenadante gerado foi filtrado em papel de filtro de $20 \mu \mathrm{m}$ e submetido às análises laboratoriais.

Com o intuito de se obter informações acerca do equilíbrio entre adsorvato (COD) e o CAP, foram feitos ensaios para se determinar a Isoterma de Adsorção.

Isoterma de adsorção representa a quantidade de um determinado soluto adsorvido por uma superfície adsorvente, em função da concentração de equilíbrio do soluto (Tagliaferro et al., 2011). Para tanto, realizou-se ensaios com o sobrenadante filtrado produzido em ensaios de coagulação/floculação com dosagens de 0,4 e de 0,6 gFe. $\mathrm{L}^{-1}$, variando-se a dosagem de CAP de 0,5 a $3 \mathrm{~g} \cdot \mathrm{L}^{-1}$ num tempo de contato de cinco dias. Concomitantemente, foi realizada uma avaliação do comportamento cinético do sistema.

\subsection{Métodos Analíticos}

As variáveis monitoradas ao longo do estudo foram: $\mathrm{pH}$, cor aparente, turbidez, condutividade 
elétrica e COD. Os métodos analíticos foram empregados de acordo com o Standard Methods for the Examination of Water and Wastewater, $21^{\text {th }}$ Edition (APHA/AWWA/WEF, 2005).

\section{DISCUSSÃO DOS RESULTADOS}

\subsection{Caracterização do Lixiviado de Aterro Sanitário}

A fim de avaliar a eficiência do tratamento, foi feita a caracterização do lixiviado utilizado ao longo do estudo. Na Tabela 1 são apresentados os valores obtidos.

Tabela 1 - Caracterização do lixiviado de aterro sanitário

\begin{tabular}{|l|l|}
\hline \multicolumn{1}{|c|}{ Variáveis } & Concentração \\
\hline Carbono orgânico dissolvido (COD) $-{\mathrm{mgC} . \mathrm{L}^{-1}}^{-1}$ & 363 \\
\hline Demanda química de oxigênio (DQQO) $-\mathrm{mgO}_{2} \mathrm{~L}^{-1}$ & 2433 \\
\hline Demanda bioquímica de oxigênio (DBO) $-\mathrm{mgO}_{2} \mathrm{~L}^{-1}$ & 402 \\
\hline Nitrogênio total kjeldahl (NKT) $-\mathrm{mgN} \mathrm{L}^{-1}$ & 1960 \\
\hline Nitrogênio amoniacal - $\mathrm{mgN}-\mathrm{NH}_{4}{ }^{+} \mathrm{L}^{-1}$ & 1680 \\
\hline Sólidos em Suspensão Totais $(\mathrm{SST})-\mathrm{mg} \mathrm{L}^{-1}$ & 1400 \\
\hline Cor aparente- UC & 12500 \\
\hline Turbidez- UT & 1278 \\
\hline Condutividade elétrica - $\mathrm{mS} . \mathrm{cm}^{-2}$ & 20,5 \\
\hline pH & 8,5 \\
\hline
\end{tabular}

A baixa relação $\mathrm{DBO} / \mathrm{DQO} O(0,16)$, o pH em torno de 8,5 e a predominância de nitrogênio amoniacal em detrimento do orgânico, permitem definir o lixiviado utilizado no estudo como proveniente de uma célula de aterro tipicamente estabilizado, onde os processos metabólicos envolvendo as substâncias orgânicas facilmente biodegradáveis já ocorreram, mantendo-se a predominância de compostos orgânicos recalcitrantes em sua composição.

\subsection{Ensaios de Tratabilidade}

\subsubsection{Ensaios de coagulação/floculação}

As Tabelas 2, 3 e 4 apresentam os resultados obtidos nessa etapa.
Tabela 2 - Resultados obtidos nos ensaios de coagulação/floculação variando a dosagem de coagulante em diferentes $\mathrm{pH}$ (Amostras decantadas)

\begin{tabular}{|c|c|c|c|c|c|c|c|c|}
\hline \multicolumn{5}{|c|}{ turbidez (UT) } & \multicolumn{4}{|c|}{ cor (UC) } \\
\hline \multicolumn{9}{|c|}{ Dosagem de coagulante $\mathrm{FeCl}_{3}-\mathrm{gFe} . \mathrm{L}^{-1}$} \\
\hline $\mathrm{pH}$ & 0,2 & 0,4 & 0,6 & 0,8 & 0,2 & 0,4 & 0,6 & 0,8 \\
\hline 3 & 20,9 & 19,2 & 8,6 & 3,9 & 620 & 520 & 260 & 248 \\
\hline 4 & 38,2 & 27,6 & 5,6 & 3,1 & 680 & 660 & 250 & 108 \\
\hline 5 & 51,5 & 48,6 & 17,4 & 5,9 & 1120 & 940 & 320 & 158 \\
\hline 7 & 198 & 202 & 171 & 35,1 & 3070 & 3180 & 1050 & 1010 \\
\hline 8 & 40 & 23,5 & 18 & 17 & 2250 & 1770 & 1500 & 1260 \\
\hline 9 & 113 & 97 & 65 & 17 & 2740 & 2050 & 1610 & 1540 \\
\hline
\end{tabular}

Tabela 3 - Resultados de turbidez e cor obtidos nos ensaios de coagulação/floculação variando a dosagem de coagulante em diferentes $\mathrm{pH}$ (Amostras filtradas)

\begin{tabular}{|l|l|l|l|l|l|l|l|l|}
\hline \multicolumn{7}{|c|}{ turbidez (UT) } & \multicolumn{5}{c|}{ cor (UC) } \\
\hline \multicolumn{7}{|c|}{ Dosagem de coagulante $\mathrm{FeCl}_{3}-$ gFe.L-1 $^{-1}$} \\
\hline $\mathrm{pH}$ & 0,2 & 0,4 & 0,6 & 0,8 & 0,2 & 0,4 & 0,6 & 0,8 \\
\hline 3 & 20 & 16 & 5 & 2 & 510 & 500 & 220 & 226 \\
\hline 4 & 36 & 25 & 3 & 1 & 650 & 605 & 223 & 81 \\
\hline 5 & 46 & 47 & 15 & 3 & 950 & 650 & 260 & 118 \\
\hline 7 & 190 & 200 & 150 & 31 & 3000 & 3100 & 1000 & 940 \\
\hline 8 & 37 & 20 & 17 & 15 & 1900 & 1660 & 1400 & 1200 \\
\hline 9 & 105 & 90 & 61 & 15 & 2550 & 1940 & 1580 & 1500 \\
\hline
\end{tabular}

Tabela 4 - Resultados de COD obtidos nos ensaios de coagulação/floculação variando a dosagem de coagulante em diferentes $\mathrm{pH}$

\begin{tabular}{|l|l|l|l|l|}
\hline \multicolumn{5}{|c|}{ COD (mgC.L-1) } \\
\hline \multicolumn{5}{|c|}{ Dosagem de coagulante $\mathrm{FeCl}_{3}-$ gFe.L-1 $^{-1}$} \\
\hline $\mathrm{pH}$ & 0,2 & 0,4 & 0,6 & 0,8 \\
\hline 3 & 295 & 255 & 161 & 117 \\
\hline 4 & 269 & 221 & 162 & 99 \\
\hline 5 & 240 & 212 & 152 & 121 \\
\hline 7 & 287 & 255 & 20 & 190 \\
\hline 8 & 300 & 298 & 254 & 231 \\
\hline 9 & 341 & 322 & 299 & 268 \\
\hline
\end{tabular}

Os resultados obtidos em meio ácido $(\mathrm{pH}$ na faixa de 3 a 5) demonstraram claramente a melhor eficiência do processo de coagulação/floculação tanto em termos de redução de COD como na remoção de cor e turbidez, sendo que para essas variáveis, não se observou uma variação significativa nos valores das amostras Decantadas e Filtradas. 
Admitindo-se que os compostos refratários podem ser quantificados indiretamente por meio da análise de COD, e sendo sua remoção o objeto principal de estudo dessa pesquisa, na Figura 1 são apresentadas as eficiências do tratamento obtidas em cada configuração proposta.

Figura 1 - Eficiência de remoção de COD em função do $\mathrm{pH}$ com diferentes dosagens de coagulante

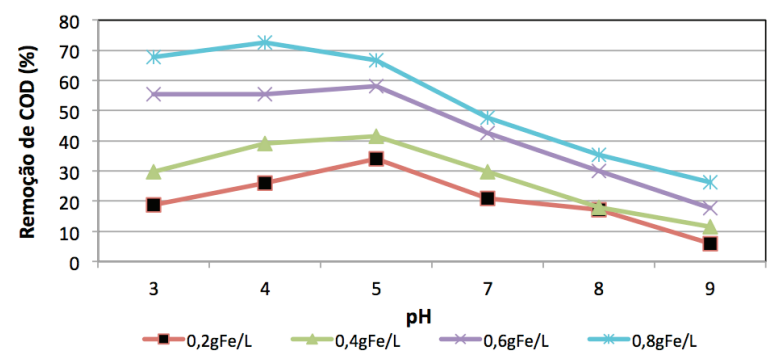

Como se pode observar, de fato, na faixa de $\mathrm{pH}$ entre 4 a 5 foram observados os melhores resultados em termos de eficiência na remoção de COD, alcançando valores da ordem de $30 \%$ a $70 \%$ em comparação ao lixiviado bruto. De acordo com Li et al. (2010), isso se deve ao fato de que as espécies formadas pela hidrólise do coagulante em meio ácido formam cátions polinucleares que apresentam uma maior afinidade aos compostos coloidais com carga negativa presentes na amostra, bem como em função do comportamento das substâncias húmicas, as quais se mantêm solúveis em meio alcalino.

Em relação ao comportamento do coagulante, buscando uma melhor relação custo-benefício, não se observa um ganho considerável de remoção de COD a partir da dosagem de $0,6 \mathrm{gFe} . \mathrm{L}^{-1}$, em $\mathrm{pH} 5$.

Um ponto importante a ressaltar é que, como era de se esperar, observou-se um aumento considerável na condutividade elétrica, quando comparado com o valor medido no lixiviado bruto, devido às altas concentrações de coagulante empregadas nos testes. Na Tabela 5 estão apresentados os resultados obtidos.

Tabela 5 - Resultados de condutividade elétrica obtidos nos ensaios de coagulação/floculação variando a dosagem de coagulante em diferentes $\mathrm{pH}$

\begin{tabular}{|l|l|l|l|l|}
\hline \multicolumn{5}{|c|}{ Condutividade elétrica ${\mathrm{mS} . \mathrm{cm}^{-2}}^{-2}$} \\
\hline \multicolumn{5}{|c|}{ Dosagem de coagulante $\mathrm{FeCl}_{3}-\mathrm{gFe} . \mathrm{L}^{-1}$} \\
\hline $\mathrm{pH}$ & 0,2 & 0,4 & 0,6 & 0,8 \\
\hline 3 & 38,4 & 36,1 & 36,7 & 41,5 \\
\hline 4 & 36,9 & 37,2 & 38,8 & 37,8 \\
\hline 5 & 34,7 & 35,4 & 34 & 36,8 \\
\hline 7 & 26,2 & 26,4 & 22,5 & 20 \\
\hline 8 & 21,2 & 19,2 & 19,1 & 19,7 \\
\hline 9 & 22,9 & 20,6 & 18,8 & 20 \\
\hline
\end{tabular}

\subsubsection{Ensaios de Adsorção}

\subsubsection{Emprego de CAP em conjunto ao coagulante}

As Tabelas 6 e 7 apresentam os resultados obtidos das variáveis cor e turbidez para as amostras filtradas geradas nos ensaios com uso combinado de coagulação/floculação e adsorção em CAP.

Tabela 6 - Resultados de cor e turbidez obtidos com o uso combinado de cloreto férrico e CAP - pH 4,5 a 5,5

\begin{tabular}{|c|c|c|c|c|c|c|c|c|c|c|c|c|}
\hline \multirow{3}{*}{ gFe. $\mathrm{L}^{-1}$} & \multicolumn{12}{|c|}{ Dosagem de CAP - gCAP.L ${ }^{-1}$} \\
\hline & \multicolumn{2}{|l|}{2} & \multicolumn{2}{|l|}{4} & \multicolumn{2}{|l|}{6} & \multicolumn{2}{|l|}{8} & \multicolumn{2}{|l|}{10} & \multicolumn{2}{|l|}{12} \\
\hline & Cor & Turb & Cor & Turb & Cor & Turb & Cor & Turb & Cor & Turb & Cor & Turb \\
\hline 0,2 & 930 & 78,6 & 808 & 69,4 & 702 & 55 & 230 & 18,3 & 205 & 17,1 & 175 & 16,2 \\
\hline 0,4 & 300 & 22,4 & 222 & 16,4 & 189 & 11,1 & 61 & 4.9 & 75 & 9,1 & 112 & 8.9 \\
\hline 0,6 & 90 & 4,3 & 59 & 4,5 & 17 & 1.7 & 36 & 2,6 & 26 & 2,3 & 34 & 3,9 \\
\hline 0,8 & 122 & 7,8 & 35 & 3 & 16 & 2 & 45 & 3 & 41 & 4 & 29 & 3 \\
\hline
\end{tabular}


Tabela 7 - Resultados de COD obtidos com o uso combinado de cloreto férrico e CAP - pH 4,5 a 5,5

\begin{tabular}{|l|l|l|l|l|l|l|}
\hline & \multicolumn{7}{|c|}{ Dosagem de CAP - gCAP.L-1 } \\
\hline gFeL $^{-1}$ & 2 & 4 & 6 & 8 & 10 & 12 \\
\hline 0,2 & 156 & 126 & 94 & 37 & 30 & 17 \\
\hline 0,4 & 106 & 73 & 59 & 21 & 16 & 15 \\
\hline 0,6 & 76 & 42 & 22 & 15 & 14 & 12 \\
\hline 0,8 & 46 & 25 & 16 & 16 & 15 & 10 \\
\hline
\end{tabular}

Como se pode observar, os resultados obtidos com o uso combinado de CAP e coagulante conferem melhores resultados quando comparados com o uso apenas do cloreto férrico no que concerne à remoção de cor, turbidez e COD.

Na Figura 2 são apresentadas as eficiências de remoção de COD obtida nos ensaios.

Figura 2 - Eficiência de remoção de COD em função da variação da aplicação conjunta de coagulante-CAP $\mathrm{pH} 4,5$ a 5,5

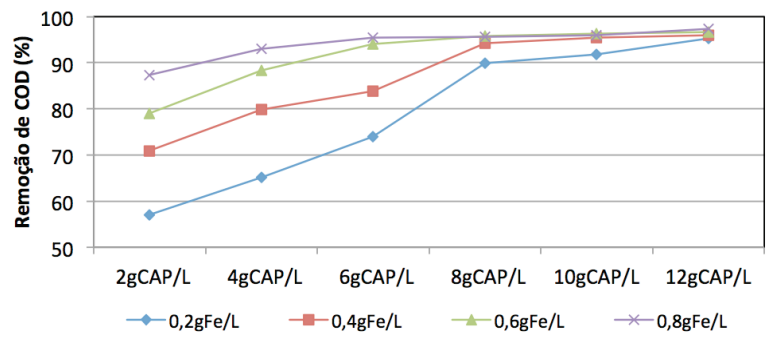

Analisando os resultados apresentados nas Tabelas 6 e 7, e na Figura 2, pode-se admitir que a dosagem de coagulante de 0,6 $\mathrm{gFe} . \mathrm{L}^{-1}$ combinada com 8 gCAP.L $\mathrm{L}^{-1}$ foi a que forneceu os melhores resultados, com eficiência de remoção de 99,7\% para cor; $99,8 \%$ para turbidez e $95,6 \%$ para COD.

Pode-se inferir que as partículas de CAP, além de possuírem a capacidade adsorvente, funcionaram como núcleo formador de floco, otimizando o processo de coagulação-floculação-sedimentação.

No que diz respeito à variável condutividade elétrica, novamente não se obteve um bom resultado final, em função das altas dosagens de coagulantes aplicadas aos testes, como se pode observar na Tabela 8.

Tabela 8 - Resultados de Condutividade elétrica obtidos com o uso combinado de cloreto férrico e CAP $-\mathrm{pH} 4,5$ a 5,5

\begin{tabular}{|c|c|c|c|c|c|c|}
\hline \multicolumn{7}{|c|}{ Dosagem de CAP - gCAP. L $^{-1}$} \\
\hline $\mathrm{gFel}^{-1}$ & $2 \mathrm{gCAP}^{\mathrm{L}} \mathrm{L}^{-1}$ & $4 \mathrm{gCAP}^{\mathrm{L}} \mathrm{L}^{-1}$ & $6 \mathrm{gCAP}^{-1} \mathrm{~L}^{-1}$ & $8 \mathrm{gCAP}^{-\mathrm{L}^{-1}}$ & 10 gCAP.L- & $12 \mathrm{gCAP}^{-\mathrm{L}^{-1}}$ \\
\hline 0,2 & 34,5 & 37,5 & 35 & 36,7 & 35,5 & 35,3 \\
\hline 0,4 & 36 & 37,7 & 34,2 & 36,4 & 34,8 & 34,2 \\
\hline 0,6 & 25,7 & 34,5 & 34,7 & 34,1 & 34,1 & 32,7 \\
\hline 0,8 & 36 & 32,2 & 31 & 34,2 & 33,9 & 30,9 \\
\hline
\end{tabular}

\subsubsection{Emprego de CAP subsequente ao Coagulante}

Nas Tabelas 9 e 10 são apresentados os resultados obtidos das variáveis cor, turbidez e COD para as amostras produzidas nesses ensaios.

Tabela 9 - Resultados de cor e turbidez obtidos mediante aplicação de CAP ao sobrenadante do processo coagulação/ floculação - pH 4,5 a 5,5

\begin{tabular}{|c|c|c|c|c|c|c|c|c|c|c|c|c|}
\hline \multicolumn{13}{|c|}{ Dosagem de CAP - gCAP.L-1 } \\
\hline \multirow[t]{2}{*}{$\mathrm{gFeL}^{-1}$} & \multicolumn{2}{|l|}{2} & \multicolumn{2}{|l|}{4} & \multicolumn{2}{|l|}{6} & \multicolumn{2}{|l|}{8} & \multicolumn{2}{|l|}{10} & \multicolumn{2}{|l|}{12} \\
\hline & Cor & Turb & Cor & Turb & Cor & Turb & Cor & Turb & Cor & Turb & Cor & Turb \\
\hline 0,2 & 505 & 6 & 162 & 5 & 84 & 3 & 284 & 2 & 121 & 2 & 84 & 2 \\
\hline 0,4 & 429 & 6 & 51 & 3,2 & 11 & 1 & 187 & 1 & 49 & 0,3 & 9 & 0,3 \\
\hline 0,6 & 107 & 2 & 16 & 0,2 & 14 & 0,3 & 18 & 0,2 & 13 & 0,4 & 15 & 0,4 \\
\hline 0,8 & 20 & 0,3 & 15 & 0,3 & 16 & 0,5 & 17 & 0,3 & 13 & 0,5 & 16 & 0,6 \\
\hline
\end{tabular}

Tabela 10 - Resultados de COD mediante aplicação de CAP ao sobrenadante do processo coagulação/floculação - pH 4,5 a 5,5

\begin{tabular}{|l|l|l|l|l|l|l|l|}
\hline \multicolumn{9}{|c|}{ Dosagem de CAP - gCAP.- } \\
\hline gFet $^{-1}$ & 2 & 4 & 6 & 8 & 10 & 12 \\
\hline 0,2 & 133 & 76 & 41 & 27 & 20 & 18 \\
\hline 0,4 & 132 & 63 & 27 & 17 & 13 & 13 \\
\hline 0,6 & 64 & 15 & 13 & 12 & 12 & 11 \\
\hline 0,8 & 29 & 15 & 13 & 11 & 10 & 9 \\
\hline
\end{tabular}


Pode-se notar uma considerável melhora nos resultados apresentados nesses ensaios quando comparados com os obtidos mediante a aplicação conjunta de coagulante/CAP.

Tal fato se deve, muito provavelmente, em função da remoção inicial de sólidos promovida pelo processo de coagulação-floculação produzir um sobrenadante com características facilitadoras para a interação entre poluente-CAP, bem como pela diminuição da interferência produzida pela presença dos íons ferro advindos do coagulante, uma vez que se observa uma forte interação entre esses e o CAP (Li et al., 2010).

Na Figura 3 estão apresentados os resultados de eficiência de remoção de COD obtidos nos ensaios.

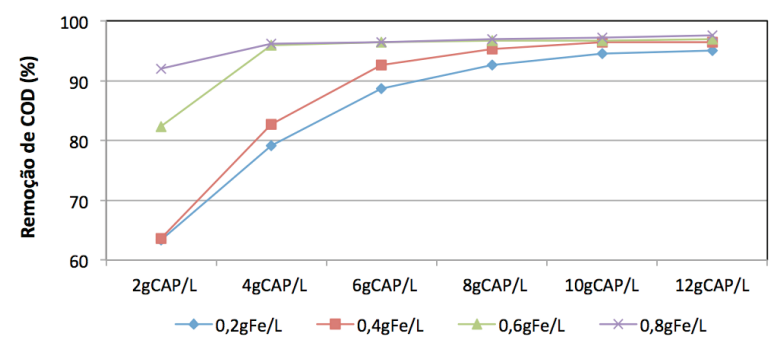

Figura 3 - Eficiência de remoção de COD em função da variação da aplicação de CAP ao sobrenadante do processo de coagulação/floculação - pH 4,5 a 5,5
De acordo com a Figura 3, pode-se admitir que a partir da dosagem de coagulante de 0,6 $\mathrm{gFe} . \mathrm{L}^{-1}$ combinada com a aplicação no pós-tratamento de 4 gCAP. $L^{-1}$ não se observam incrementos significativos de remoção de COD, o que permite inferir que tal combinação foi a que produziu os melhores resultados, com eficiência de remoção de $99,9 \%$ para cor; $99,9 \%$ para turbidez e $96,1 \%$ para COD.

A aplicação de CAP ao sobrenadante advindo do processo de coagulação-floculação, como uma etapa de pós-tratamento, demonstra, portanto, para esses ensaios, uma redução de $50 \%$ no emprego de CAP quando comparado ao uso conjunto do coagulante/CAP, o que confirma a tese de que essa configuração parece ser a mais promissora para esse tipo de tratamento.

No que diz respeito à condutividade elétrica, observou-se o mesmo fenômeno já descrito nos demais ensaios. Na Tabela 11 estão apresentados os resultados.

Tabela 11 - Resultados obtidos de condutividade elétrica mediante aplicação de CAP ao sobrenadante do processo coagulação/floculação.

\begin{tabular}{|l|l|l|l|l|l|l|}
\hline \multicolumn{7}{|c|}{ Dosagem de CAP - gCAP. $\mathbf{L}^{-1}$} \\
\hline gFeL $^{-1}$ & 2 & 4 & 6 & 8 & 10 & 12 \\
\hline 0,2 & 42,2 & 43,1 & 42,7 & 43,4 & 39,6 & 43,7 \\
\hline 0,4 & 43,8 & 41,8 & 42 & 42,2 & 41,4 & 41,6 \\
\hline 0,6 & 42,5 & 42 & 42,6 & 42,7 & 42,5 & 42,5 \\
\hline 0,8 & 40,4 & 40,4 & 40,2 & 40,5 & 40,3 & 40,6 \\
\hline
\end{tabular}

\subsubsection{Isoterma de Adsorção}

Na Tabela 12 são apresentados os valores obtidos ao término dessa etapa para cada dosagem e condição avaliada.

Tabela 13 - Resultados iniciais obtidos nos ensaios para determinação da isoterma de adsorção

\begin{tabular}{|l|l|l|l|l|}
\multicolumn{5}{|c|}{ Condições iniciais - sobrenadante } \\
\hline Doasagens & $\mathrm{pH}$ & $\operatorname{cor}(\mathrm{UC})$ & turbidez (NTU) & COD $\left(\mathrm{mgC} . \mathrm{L}^{-1}\right)$ \\
\hline $0,4 \mathrm{gFe} \mathrm{L}^{-1}$ & 5,4 & 444 & 20,7 & 149 \\
\hline $0,6 \mathrm{gFe} \mathrm{L}^{-1}$ & 5,1 & 123 & 4,1 & 108 \\
\hline
\end{tabular}


Em seguida, foram transferidas alíquotas de 100 $\mathrm{mL}$ de cada sobrenadante para erlenmeyers, e adicionadas em cada um, dosagens de CAP de 0,5; 1,$0 ; 1,5 ; 2,0 ; 2,5$ e 3,0 gCAP.L $L^{-1}$, para que, posteriormente, fossem tampados e mantidos em agitação por cinco dias a 150 rpm.
Ao término do período, o conteúdo de cada erlenmeyer foi devidamente filtrado em $20 \mu \mathrm{m}$ e analisado. Na Tabela 13 são apresentados os valores obtidos.

Tabela 14 - Resultados finais obtidos nos ensaios para determinação da isoterma de adsorção

\begin{tabular}{|c|c|c|c|c|c|c|c|}
\hline \multirow[t]{2}{*}{ erly } & \multirow{2}{*}{$\begin{array}{l}\text { CAP } \\
g \cdot \mathrm{L}^{-1}\end{array}$} & \multicolumn{2}{|c|}{ COD $\left(m g C . L^{-1}\right)$} & \multicolumn{2}{|c|}{$\operatorname{cor}(U C)$} & \multicolumn{2}{|c|}{ turbidez (NTU) } \\
\hline & & $0,4 \mathrm{gFe} \cdot \mathrm{L}^{-1}$ & $0,6 \mathrm{gFe} \cdot \mathrm{L}^{-1}$ & $0,4 \mathrm{gFe} . \mathrm{L}^{-1}$ & $0,6 \mathrm{gFe} . \mathrm{L}^{-1}$ & $0,4 \mathrm{gFe} \cdot \mathrm{L}^{-1}$ & $0,6 \mathrm{gFe} \cdot \mathrm{L}^{-1}$ \\
\hline 1 & 0,5 & 105 & 83 & 313 & 71 & 17 & 1,5 \\
\hline 2 & 1 & 91 & 55 & 308 & 40 & 18 & 1,3 \\
\hline 3 & 1,5 & 74 & 37 & 276 & 20 & 18 & 0,8 \\
\hline 4 & 2 & 55 & 26 & 101 & 19 & 5 & 1 \\
\hline 5 & 2,5 & 38 & 22 & 53 & 22 & 2 & 1,1 \\
\hline 6 & 3 & 25 & 19 & 22 & 22 & 1 & 1,4 \\
\hline
\end{tabular}

Com os resultados iniciais e finais de COD para cada ensaio, foi possível aplicar o modelo empírico proposto por Freundlich, a fim de avaliar e prever as condições de equilíbrio entre adsorvato (COD) e adsorvente (CAP).

A expressão proposta por Freundlich é representada pelas equações (01) e (02):

$\mathrm{q}_{\mathrm{e}}=\mathrm{K}_{\mathrm{ad}} * \mathrm{C}_{\mathrm{e}}^{1 / \mathrm{n}} \quad$ Equação (01)

ou:

$\log \mathrm{q}_{\mathrm{e}}=\log \mathrm{K}_{\mathrm{ad}}+1 / \mathrm{n} \log \mathrm{C}_{\mathrm{e}}$ Equação (02)

Onde:

$\mathrm{q}_{\mathrm{e}}$ : quantidade do adsorvato por unidade de adsorvente $\left(\mathrm{mg}^{\mathrm{g}} \mathrm{g}^{-1}\right)$

$\mathrm{C}_{\mathrm{e}}$ : concentração do adsorvato no equilíbrio $\left(\mathrm{mg} \cdot \mathrm{L}^{-1}\right)$

$\mathrm{K}_{\mathrm{ad}}$ e $\mathrm{n}$ : coeficientes a serem determinados empiricamente. $\mathrm{K}_{\mathrm{ad}}$ está relacionado à capacidade de adsorção do adsorvato pelo adsorvente, enquanto n depende das características da adsorção.

Na Figura 4 está representado o comportamento ocorrido em cada ensaio, e na Tabela 15 estão apresentadas as constantes obtidas após a aplicação do modelo de Freundlich.

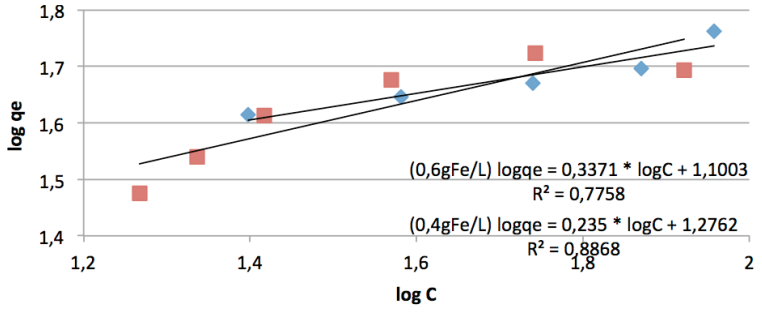

$\bullet 0,4 \mathrm{gFe} / \mathrm{L} \quad \square, 6 \mathrm{gFe} / \mathrm{L}$

Figura 4 - Comportamento observado entre adsorvato-adsorvente para determinação da isoterma de adsorção

Tabela 14 - Constantes de equilíbrio entre adsorvente-adsorvato.

\begin{tabular}{|l|l|l|l|}
\hline Dosagem de Coagulante & $\mathrm{K}$ & $1 / \mathrm{n}$ & $\mathrm{R}^{2}$ \\
\hline $0,4 \mathrm{gFe} . \mathrm{L}^{-1}$ & 1,1003 & 0,3371 & 0,78 \\
\hline $0,6 \mathrm{gFe} . \mathrm{L}^{-1}$ & 1,2762 & 0,235 & 0,89 \\
\hline
\end{tabular}

A análise da Figura 4 e dos resultados das constantes obtidas em cada ensaio demonstra que os comportamentos para as duas condições estudadas resultaram semelhantes, do que se conclui que a capacidade de adsorção do COD pelo CAP independe da dosagem de coagulante, sendo que o processo é preferencialmente regido pela afinidade existente entre os componentes presentes no lixiviado (adsorvato) e o CAP (adsorvente).

Para investigar o tempo mínimo de contato exigido para a máxima remoção do adsorvato pelo CAP, foi realizado um novo ensaio com dosagem 
de 0,4 gFe.L-1 seguido de aplicação de $4 \mathrm{gCAP} \cdot \mathrm{L}^{-1}$ ao sobrenadante. Foram feitas retiradas de alíquotas de 10 em 10 minutos, filtradas e determinado o COD. $\mathrm{Na}$ Figura 5 são lançados em gráfico os resultados obtidos.

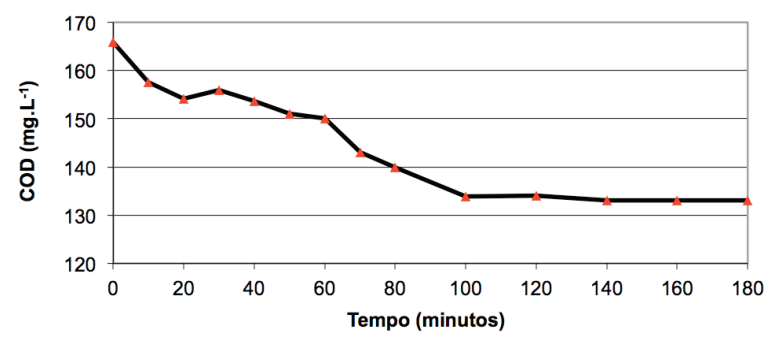

Figura 5 - Comportamento cinético do processo de adsorção entre adsorvato/adsorvente

De acordo com a Figura 5, o equilíbrio entre adsorvato e CAP pode ser obtido praticamente por volta de 90 minutos de tempo de contato, valor igual ao encontrado por Li et al. (2010) em seus estudos empregando sais de alumínio como coagulante em conjunto com CAP. Dessa forma, os resultados dos testes de adsorção ao longo da pesquisa, mantidos num tempo de contato de três horas, garantem que em cada situação, o ponto de equilíbrio cinético já estava satisfeito, garantindo que os valores medidos estão condizentes com a capacidade de adsorção do CAP utilizado.

\section{CONCLUSÃO}

Ao término deste trabalho, foi possível concluir que: O uso combinado de coagulante e carvão ativado em pó demonstrou ser um método promissor para tratamento de lixiviados de aterro sanitários estabilizados no que diz respeito à remoção de COD, cor e turbidez; Em cada configuração deve-se buscar as dosagens de CAP e coagulante que levem a uma melhor relação custo-benefício;

A faixa de $\mathrm{pH}$ ácida demostrou ser adequada para a ação do coagulante cloreto férrico sobre o lixiviado;

A aplicação de CAP como pós-tratamento do processo de coagulação-floculação mostrou-se mais vantajosa do que quando dosado em conjunto ao coagulante;

O tempo de adsorção mostrou-se ser um parâmetro fundamental para a otimização do processo.

\section{REFERÊNCIAS BIBLIOGRÁFICAS}

APHA/AWWA/WEF. Standard Methods for the Examination of Water and Wastewater. 21 ${ }^{\mathrm{a}}$ Edição. Washington D.C.: 2005.

CAMPOS, J.R. Descarte de Lixividado de Aterro Sanitário em Estações de Tratamento de Esgoto: Uma Análise Crítica. São Paulo: Revista DAE, vol. 62 número 197, p. 6-17, 2014.

ÇECEN, F. , ÇAKIROLUM, D. Impact of Landifill Leachate on the CoTreatment of Domestic Wastewater. Biotechnology Letters 23, [S.L.] p. 821-826, 2001.

CHRISTENSEN, J. B.; BJERG, P. P. L.; JENSEN, D. L.; CHRISTENSEN, A.; BAUM, A.; ALBRECHTSEN, H. J.; HERON, G. Biochemistry of Landfill Leachate plumes - Applied Geochemistry, [S.L.], v. 16, p. 659-718, 2001.

KJELDSEN, P.; BARLAZ, M. A.; ROOKER, A. P.; BAUN, A.; LEDIN, A.; CHRISTENSEN, A. Present and Long-Term Composition of MSW Landfill Leachate: A Review - Environmental Science and Technology, [S.L.], v. 32, p. 297-336, 2002.

KURNIAWAN, T. A.; WAI-HUNG LO; YS CHAN, G., Physico-Chemical Treatments for Removal of Recalcitrant Contaminants from Landfill Leachate - Journal of Hazardous Materials, ELSEVIER, p. 80-100, 2005.

LI, W.; HUA, T.; ZHOU, Q.; ZHANG, S.; LI, F. Treatment of Stabilized Landfill Leachate by the Combined Process of Coagulation/ Flocculation and Powder Activated Carbon Adsorption - Journal of Desalinisation, [S.L.], p. 56-62, 2010.

MORAVIA, W. G.; AMARAL, M. C. S.; FERREIRA, C. F. A.; LANGE, L. C.; MARTINS, L. K. Estudo de Diferentes Métodos de Partida de Sistemas de Tratamento Aeróbio para Lixiviados de Aterro Sanitário Estabilizado em Escala de Laboratório, Anais do $24^{\circ}$ Congresso Brasileiro de Engenharia Sanitária e Ambiental. [S.L.]: [S.D.].

QASIM, S.R; CHIANG, W. Sanitary Landfill Leachate - Generation, Control and Treatment. Technomic Publication, Pensilvânia, EUA, 1994.

RENOU, S; GIVAUDAN, J.G.; POULAIN, S.; DIRASSOUYAN, F.; MOULIN, P. Landfill Leachate Treatment: Review and Opportunity, Journal of $\mathrm{Ha}-$ zardous Materials. ELSEVIER, 468 - 493p., 2007.

SILVA, R.C.A. Pós-Tratamento de Sistema Biológico Aeróbio em Batelada de Lixiviado de Aterro Sanitário Através de Processos FísicoQuímicos. Dissertação apresentada na Escola Politécnica da USP. São Paulo: 2011

SOUTO, G.D.B.; POVINELLI, J. Características do Lixiviado de Aterros Sanitários no Brasil, $24^{\circ}$ Congresso Brasileiro de Engenharia Sanitária e Ambiental, 2005.

TAGLIAfERro, GV., et al. Adsorção de Chumbo, Cádmio e Prata em Óxido de Nióbio (v) Hidratado Preparado pelo Método da Precipitação em Solução Homogênea. Química Nova, [S.L], v. 34, n. 1, p. 101105, 2011.

WISZNIOWSK, J.; ROBERT, D.; SURMACZ-GORSKA, J.; MIKSCH, K.; WEBER, J.V. Landfill Leachate Treatment Methods: A Review. Environ. Chem. Lett., [S.L.], p. 51-61, 2005. 